УДК 512.554.1

\title{
The variety of nilpotent Tortkara algebras
}

\author{
Ilya B. Gorshkov* \\ Institute of Mathematics and Computer Science \\ Siberian Federal University \\ Svobodny, 79, Krasnoyarsk, 660041 \\ Russia \\ Ivan Kaygorodov ${ }^{\dagger}$ \\ CMCC, Universidade Federal do ABC \\ Santo André, Brasil \\ Alexey A. Kytmanov $\ddagger$ \\ Institute of Space and Information Technologies \\ Siberian Federal University \\ Kirensky, 26, Krasnoyarsk, 660074 \\ Russia \\ Mohamed A. Salim \\ United Arab Emirates University \\ Al Ain, UAE
}

Received 06.11.2018, received in revised form 06.12.2018, accepted 06.08.2018

We give algebraic and geometric classification of all 5-dimensional nilpotent Tortkara algebras over complex field.

Keywords: Tortkara algebra, algeraic classification, central extension, geometric classification, degenerations.

DOI: 10.17516/1997-1397-2019-12-2-173-184.

The algebraic classification (up to isomorphism) of algebras of dimension $n$ from a certain variety defined by some family of polynomial identities is a classic problem in the theory of non-associative algebras. There are many results related to algebraic classification of small dimensional algebras in varieties of Jordan, Lie, Leibniz, Zinbiel and many other algebras [16-19, $25,28,37]$. Another interesting direction in classifications of algebras is a geometric classification. There are many results related to geometric classification of Jordan, Lie, Leibniz, Zinbiel and many other algebras $[3-6,8,9,11,12,29-33,35-38,41]$. In the present paper, we give algebraic and geometric classifications of nilpotent algebras of a new class of non-associative algebras introduced by Dzhumadildaev in [21].

Zinbiel algebras were introduced by Loday in [39]. They were studied in [15,20,22, 23,35,40, 44]. Under the Koszul duality, the operad of Zinbiel algebras is dual to the operad of Leibniz algebras. Zinbiel algebras are related to Tortkara algebras [21] and Tortkara triple systems [10].

An algebra $\mathbf{A}$ is called a Zinbiel algebra if it satisfies the identity

$$
(x y) z=x(y z+z y) .
$$

Every Zinbiel algebra with the commutator multiplication defines a Tortkara algebra. An anticommutative algebra $\mathbf{A}$ is called a Tortkara algebra if it satisfies the identity

$$
(a b)(c b)=J(a, b, c) b, \text { where } J(a, b, c)=(a b) c+(b c) a+(c a) b .
$$

*ilygor8@gmail.com

$\dagger$ kaygorodov.ivan@gmail.com

‡aakytm@gmail.com

$\S$ msalim@uaeu.ac.ae

(c) Siberian Federal University. All rights reserved 
It is easy to see that every metabelian Lie algebra (i.e., $(x y)(z t)=0)$ is a Tortkara algebra and every Dual Mock-Lie algebra (i.e., antiassociative and anticommutative) is a Tortkara algebra.

Our method of classification of nilpotent Tortkara algebras is based on calculation of central extensions of smaller nilpotent algebras from the same variety. Central extensions play an important role in quantum mechanics: one of the earlier encounters is by means of Wigner's theorem which states that symmetry of a quantum mechanical system determines an (anti-)unitary transformation of a Hilbert space. Another area of physics where one encounters central extensions is the quantum theory of conserved currents of a Lagrangian. These currents span an algebra which is closely related to so called affine Kac-Moody algebras, which are the universal central extension of loop algebras. Central extensions are needed in physics, because the symmetry group of a quantized system usually is a central extension of the classical symmetry group, and in the same way, the corresponding symmetry Lie algebra of the quantum system is, in general, a central extension of the classical symmetry algebra. Kac-Moody algebras have been conjectured to be a symmetry groups of a unified superstring theory. The centrally extended Lie algebras play a dominant role in quantum field theory, particularly in conformal field theory, string theory and in $M$-theory. In the theory of Lie groups, Lie algebras and their representations, a Lie algebra extension is an enlargement of a given Lie algebra $g$ by another Lie algebra $h$. Extensions arise in several ways. There is a trivial extension obtained by taking a direct sum of two Lie algebras. Other types are split extension and central extension. Extensions may arise naturally, for instance, when forming a Lie algebra from projective group representations. A central extension and an extension by a derivation of a polynomial loop algebra over finite-dimensional simple Lie algebra give a Lie algebra which is isomorphic to a non-twisted affine Kac-Moody algebra [7, Chapter 19]. Using the centrally extended loop algebra one may construct a current algebra in two spacetime dimensions. The Virasoro algebra is the universal central extension of the Witt algebra, the Heisenberg algebra is the central extension of a commutative Lie algebra [7, Chapter 18]. The algebraic study of central extensions of Lie and non-Lie algebras has a very rich history $[2,26,27,34,42,43,45]$. In particular, Skjelbred and Sund used central extensions of Lie algebras for a classification of nilpotent Lie algebras [42]. Following that, there were described all non-Lie central extensions of all 4-dimensional Malcev algebras [27], all non-associative central extensions of 3-dimensional Jordan algebras [26], all anticommutative central extensions of 3-dimensional anticommutative algebras [13], and all central extensions of 2-dimensional algebras [14] by means of the method described by Skjelbred and Sund. We also note that the method of central extensions is an important tool in the classification of nilpotent algebras (see, e.g., [24]). Using this method, there were described all 4-dimensional nilpotent associative algebras [18], all 5-dimensional nilpotent Jordan algebras [25], all 5-dimensional nilpotent restricted Lie agebras [17], all 6-dimensional nilpotent Lie algebras [16,19], all 6-dimensional nilpotent Malcev algebras [28], and some others.

\section{The algebraic classification of 5-dimensional nilpotent Tortkara algebras}

\subsection{Preliminaries}

\section{A method for classification of nilpotent algebras}

Throughout this paper, we use the notations and methods introduced in [14,26,27] and adapted for the Tortkara case with some modifications. Below we give some of the most important definitions.

Let $(\mathbf{A}, \cdot)$ be a Tortkara algebra over an arbitrary base field $\mathbf{k}$ of characteristic not 2 and $\mathbb{V}$ a vector space over the same base field $\mathbf{k}$. Then the $\mathbf{k}$-linear space $Z^{2}(\mathbf{A}, \mathbb{V})$ is defined as the 
set of all skew-symmetric bilinear maps $\theta: \mathbf{A} \times \mathbf{A} \longrightarrow \mathbb{V}$ such that

$$
\theta(x y, z t)+\theta(x t, z y)=\theta(J(x, y, z), t)+\theta(J(x, t, z), y)
$$

Its elements are called cocycles. For a linear map $f: A \longrightarrow \mathbb{V}$, we define $\delta f: \mathbf{A} \times \mathbf{A} \longrightarrow \mathbb{V}$ by $\delta f(x, y)=f(x y)$ so that $\delta f \in Z^{2}(\mathbf{A}, \mathbb{V})$. Define $B^{2}(\mathbf{A}, \mathbb{V})=\{\theta=\delta f: f \in \operatorname{Hom}(\mathbf{A}, \mathbb{V})\}$. One can easily check that $B^{2}(\mathbf{A}, \mathbb{V})$ is a linear subspace of $Z^{2}(\mathbf{A}, \mathbb{V})$ whose elements are called coboundaries. We define the second cohomology space $H^{2}(\mathbf{A}, \mathbb{V})$ as the quotient space $Z^{2}(\mathbf{A}, \mathbb{V}) / B^{2}(\mathbf{A}, \mathbb{V})$.

Let $\operatorname{Aut}(\mathbf{A})$ be the automorphism group of the Tortkara algebra $\mathbf{A}$ and let $\phi \in \operatorname{Aut}(\mathbf{A})$. For $\theta \in Z^{2}(\mathbf{A}, \mathbb{V})$ define $\phi \theta(x, y)=\theta(\phi(x), \phi(y))$. Then $\phi \theta \in Z^{2}(\mathbf{A}, \mathbb{V})$. So, Aut $(\mathbf{A})$ acts on $Z^{2}(\mathbf{A}, \mathbb{V})$. It is easy to verify that $B^{2}(\mathbf{A}, \mathbb{V})$ is invariant under the action of Aut $(\mathbf{A})$ and so we have that $\operatorname{Aut}(\mathbf{A})$ acts on $H^{2}(\mathbf{A}, \mathbb{V})$.

Let $\mathbf{A}$ be a Tortkara algebra of dimension $m<n$ over an arbitrary base field $\mathbf{k}$ of characteristic not 2 , and $\mathbb{V}$ be a $\mathbf{k}$-vector space of dimension $n-m$. For any $\theta \in Z^{2}(\mathbf{A}, \mathbb{V})$, define the bilinear product " $[-,-]_{\mathbf{A}_{\theta}}$ " on the linear space $\mathbf{A}_{\theta}:=\mathbf{A} \oplus \mathbb{V}$ by $\left[x+x^{\prime}, y+y^{\prime}\right]_{\mathbf{A}_{\theta}}=x y+\theta(x, y)$ for all $x, y \in \mathbf{A}, x^{\prime}, y^{\prime} \in \mathbb{V}$. The algebra $\mathbf{A}_{\theta}$ is a Tortkara algebra which is called an $(n-m)$-dimensional central extension of $\mathbf{A}$ by $\mathbb{V}$. Indeed, clearly, $\mathbf{A}_{\theta}$ is a Tortkara algebra if and only if $\theta \in Z^{2}(\mathbf{A}, \mathbb{V})$.

We also call the set $\operatorname{Ann}(\theta)=\{x \in \mathbf{A}: \theta(x, \mathbf{A})=0\}$ the annihilator of $\theta$. Recall that the annihilator of an algebra $\mathbf{A}$ is defined as the ideal $\operatorname{Ann}(\mathbf{A})=\{x \in \mathbf{A}: x \mathbf{A}=0\}$, and observe that $\operatorname{Ann}\left(\mathbf{A}_{\theta}\right)=\operatorname{Ann}(\theta) \cap \operatorname{Ann}(\mathbf{A}) \oplus \mathbb{V}$.

We now state the following key result:

Lemma 1.1. Let $\mathbf{A}$ be an $n$-dimensional Tortkara algebra with $\operatorname{dim}(\operatorname{Ann}(\mathbf{A}))=m \neq 0$. Then, there exists (up to an isomorphism) a unique $(n-m)$-dimensional Tortkara algebra $\mathbf{A}^{\prime}$ and a bilinear map $\theta \in Z^{2}(\mathbf{A}, \mathbb{V})$ with $\operatorname{Ann}(\mathbf{A}) \cap \operatorname{Ann}(\theta)=0$, where $\mathbb{V}$ is a vector space of dimension $m$ such that $\mathbf{A} \cong \mathbf{A}_{\theta}^{\prime}$ and $\mathbf{A} / \operatorname{Ann}(\mathbf{A}) \cong \mathbf{A}^{\prime}$.

Proof. Let $\mathbf{A}^{\prime}$ be a linear complement of $\operatorname{Ann}(\mathbf{A})$ in $\mathbf{A}$. Define a linear map $P: \mathbf{A} \longrightarrow \mathbf{A}^{\prime}$ by $P(x+v)=x$ for $x \in \mathbf{A}^{\prime}$ and $v \in \operatorname{Ann}(\mathbf{A})$ and define a multiplication on $\mathbf{A}^{\prime}$ by $[x, y]_{\mathbf{A}^{\prime}}=P(x y)$ for $x, y \in \mathbf{A}^{\prime}$. Then, for $x, y \in \mathbf{A}$,

$$
P(x y)=P((x-P(x)+P(x))(y-P(y)-P(y)))=P(P(x) P(y))=[P(x), P(y)]_{\mathbf{A}^{\prime}} .
$$

Since $P$ is a homomorphism, $P(\mathbf{A})=\mathbf{A}^{\prime}$ is a Tortkara algebra and $\mathbf{A} / \operatorname{Ann}(\mathbf{A}) \cong \mathbf{A}^{\prime}$, which gives us the uniqueness. Now, define the map $\theta: \mathbf{A}^{\prime} \times \mathbf{A}^{\prime} \longrightarrow \operatorname{Ann}(\mathbf{A})$ by $\theta(x, y)=x y-[x, y]_{\mathbf{A}^{\prime}}$. Thus, $\mathbf{A}_{\theta}^{\prime}$ is $\mathbf{A}$ and therefore $\theta \in Z^{2}(\mathbf{A}, \mathbb{V})$ and $\operatorname{Ann}(\mathbf{A}) \cap \operatorname{Ann}(\theta)=0$. The lemma is proved.

However, in order to solve the isomorphism problem, we need to study the action of Aut (A) on $H^{2}(\mathbf{A}, \mathbb{V})$. To do that, fix $e_{1}, \ldots, e_{s}$, a basis of $\mathbb{V}$, and $\theta \in Z^{2}(\mathbf{A}, \mathbb{V})$. Then, there is a unique representation $\theta(x, y)=\sum_{i=1}^{s} \theta_{i}(x, y) e_{i}$ with $\theta_{i} \in Z^{2}(\mathbf{A}, \mathbf{k})$. Moreover, $\operatorname{Ann}(\theta)=\operatorname{Ann}\left(\theta_{1}\right) \cap$ $\operatorname{Ann}\left(\theta_{2}\right) \cap \cdots \cap \operatorname{Ann}\left(\theta_{s}\right)$. Furthermore, $\theta \in B^{2}(\mathbf{A}, \mathbb{V})$ if and only if all $\theta_{i} \in B^{2}(\mathbf{A}, \mathbf{k})$.

Given a Tortkara algebra $\mathbf{A}$, if $\mathbf{A}=I \oplus \mathbf{k x}$ is a direct sum of two ideals, then $\mathbf{k x}$ is called an annihilator component of $\mathbf{A}$.

Definition 1. A central extension of an algebra A without annihilator component is called a non-split central extension.

It is not difficult to show (see [27, Lemma 13]), that given a Tortkara algebra $\mathbf{A}_{\theta}$, if use the respresentation $\theta(x, y)=\sum_{i=1}^{s} \theta_{i}(x, y) e_{i} \in Z^{2}(\mathbf{A}, \mathbb{V})$, and $\operatorname{Ann}(\theta) \cap \operatorname{Ann}(\mathbf{A})=0$, then $\mathbf{A}_{\theta}$ has an annihilator component if and only if $\left[\theta_{1}\right],\left[\theta_{2}\right], \ldots,\left[\theta_{s}\right]$ are linearly dependent in $H^{2}(\mathbf{A}, \mathbf{k})$. 
Let $\mathbb{V}$ be a finite-dimensional vector space over k. The $\operatorname{Grassmannian} G_{k}(\mathbb{V})$ is a set of all $k$-dimensional linear subspaces of $\mathbb{V}$. Let $G_{s}\left(H^{2}(\mathbf{A}, \mathbf{k})\right)$ be the Grassmannian of subspaces of dimension $s$ in $H^{2}(\mathbf{A}, \mathbf{k})$. There is a natural action of $\operatorname{Aut}(\mathbf{A})$ on $G_{s}\left(H^{2}(\mathbf{A}, \mathbf{k})\right)$. Let $\phi \in$ $\operatorname{Aut}(\mathbf{A})$. For $W=\left\langle\left[\theta_{1}\right],\left[\theta_{2}\right], \ldots,\left[\theta_{s}\right]\right\rangle \in G_{s}\left(H^{2}(\mathbf{A}, \mathbf{k})\right)$ define $\phi W=\left\langle\left[\phi \theta_{1}\right],\left[\phi \theta_{2}\right], \ldots,\left[\phi \theta_{s}\right]\right\rangle$. Then $\phi W \in G_{s}\left(H^{2}(\mathbf{A}, \mathbf{k})\right)$. Denote the orbit of $W \in G_{s}\left(H^{2}(\mathbf{A}, \mathbf{k})\right)$ under the action of Aut $(\mathbf{A})$ by $\operatorname{Orb}(W)$. Since given

$$
W_{1}=\left\langle\left[\theta_{1}\right],\left[\theta_{2}\right], \ldots,\left[\theta_{s}\right]\right\rangle, W_{2}=\left\langle\left[\vartheta_{1}\right],\left[\vartheta_{2}\right], \ldots,\left[\vartheta_{s}\right]\right\rangle \in G_{s}\left(H^{2}(\mathbf{A}, \mathbf{k}),\right)
$$

we easily obtain that if $W_{1}=W_{2}$, then $\bigcap_{i=1}^{s} \operatorname{Ann}\left(\theta_{i}\right) \cap \operatorname{Ann}(\mathbf{A})=\bigcap_{i=1}^{s} \operatorname{Ann}\left(\vartheta_{i}\right) \cap \operatorname{Ann}(\mathbf{A})$ so that we can introduce the set

$$
T_{s}(\mathbf{A})=\left\{W=\left\langle\left[\theta_{1}\right],\left[\theta_{2}\right], \ldots,\left[\theta_{s}\right]\right\rangle \in G_{s}\left(H^{2}(\mathbf{A}, \mathbf{k})\right): \stackrel{\cap}{s}_{i=1}^{s} \operatorname{Ann}\left(\theta_{i}\right) \cap \operatorname{Ann}(\mathbf{A})=0\right\},
$$

which is stable under the action of $\operatorname{Aut}(\mathbf{A})$.

Now, let $\mathbb{V}$ be a $s$-dimensional linear space. By $E(\mathbf{A}, \mathbb{V})$ we denote the set of all non-split $s$-dimensional central extensions of $\mathbf{A}$ by $\mathbb{V}$. Then,

$$
E(\mathbf{A}, \mathbb{V})=\left\{\mathbf{A}_{\theta}: \theta(x, y)=\sum_{i=1}^{s} \theta_{i}(x, y) e_{i} \text { and }\left\langle\left[\theta_{1}\right],\left[\theta_{2}\right], \ldots,\left[\theta_{s}\right]\right\rangle \in T_{s}(\mathbf{A})\right\} .
$$

We now state the following result which can be proved as [27, Lemma 17].

Lemma 1.2. Let $\mathbf{A}_{\theta}, \mathbf{A}_{\vartheta} \in E(\mathbf{A}, \mathbb{V})$. Suppose that $\theta(x, y)=\sum_{i=1}^{s} \theta_{i}(x, y) e_{i}$ and $\vartheta(x, y)=$ $=\sum_{i=1}^{s} \vartheta_{i}(x, y) e_{i}$. Then the Tortkara algebras $\mathbf{A}_{\theta}$ and $\mathbf{A}_{\vartheta}$ are isomorphic if and only if

$$
\operatorname{Orb}\left\langle\left[\theta_{1}\right],\left[\theta_{2}\right], \ldots,\left[\theta_{s}\right]\right\rangle=\operatorname{Orb}\left\langle\left[\vartheta_{1}\right],\left[\vartheta_{2}\right], \ldots,\left[\vartheta_{s}\right]\right\rangle
$$

Consequently, there exists a one-to-one correspondence between the set of Aut (A)-orbits on $T_{s}(\mathbf{A})$ and the set of isomorphism classes of $E(\mathbf{A}, \mathbb{V})$. We therefore have the following procedure that allows us, given the Tortkara algebras $\mathbf{A}^{\prime}$ of dimension $n$, to construct all non-split central extensions of $\mathbf{A}^{\prime}$.

\section{Procedure}

1. For a given Tortkara algebra $\mathbf{A}^{\prime}$ of dimension $n$, determine $H^{2}\left(\mathbf{A}^{\prime}, \mathbf{k}\right)$ and $\operatorname{Aut}\left(\mathbf{A}^{\prime}\right)$.

2. Determine the set of $\operatorname{Aut}\left(\mathbf{A}^{\prime}\right)$-orbits on $T_{s}\left(\mathbf{A}^{\prime}\right)$.

3. For each orbit, construct the non-split Tortkara algebra corresponding to its representative.

\section{Notations}

We now introduce some notations. Let $\mathbf{A}$ be a Tortkara algebra with a basis $e_{1}, e_{2}, \ldots, e_{n}$. Then, by $\Delta_{i j}$ we denote the Tortkara bilinear form $\Delta_{i j}: \mathbf{A} \times \mathbf{A} \longrightarrow \mathbf{k}$ with $\Delta_{i j}\left(e_{l}, e_{m}\right)=0$ if $\{i, j\} \neq\{l, m\}$ and $\Delta_{i j}\left(e_{i}, e_{j}\right)=-\Delta_{i j}\left(e_{j}, e_{i}\right)=1$. Then, the set $\left\{\Delta_{i j}: 1 \leqslant i<j \leqslant n\right\}$ is a basis for the linear space of bilinear forms on $\mathbf{A}$. Then, every $\theta \in Z^{2}(\mathbf{A}, \mathbb{V})$ can be uniquely written as $\theta=\sum_{1 \leqslant i<j \leqslant n} c_{i j} \Delta_{i j}$, where $c_{i j} \in \mathbf{k}$. We use the following notations as well:

$$
\begin{array}{lll}
\mathbb{T}_{j}^{i} & - & j \text { th } i \text {-dimensional Tortkara algebra, } \\
\mathfrak{N}_{i} & - & i \text {-dimensional algebra with zero product, } \\
(\mathbf{A})_{i, j} \quad- & j \text { th } i \text {-dimensional central extension of } \mathbf{A} .
\end{array}
$$




\section{Central extensions of nilpotent low dimensional Tortkara algebras}

There are no nontrivial 1- and 2-dimensional nilpotent Tortkara algebras. There is only one nontrivial 3-dimensional nilpotent Tortkara algebra (it is the non-split central extention of $\mathfrak{N}_{2}$, see $\mathbb{T}_{01}^{3}$ below). Thanks to [13], we have a description of all anticommutative central extensions of 3-dimensional anticommutative nilpotent (Tortkara) algebras:

$$
\begin{aligned}
& \mathbb{T}_{01}^{3} \quad: \quad\left(\mathfrak{N}_{2}\right)_{3,1} \quad: \quad e_{1} e_{2}=e_{3}, \\
& \mathbb{T}_{02}^{4}:\left(\mathbb{T}_{01}^{3}\right)_{4,1} \quad: \quad e_{1} e_{2}=e_{3}, \quad e_{1} e_{3}=e_{4}, \\
& \mathbb{T}_{03}^{5} \quad: \quad\left(\mathfrak{N}_{3}\right)_{5,1} \quad: \quad e_{1} e_{2}=e_{4}, \quad e_{1} e_{3}=e_{5}, \\
& \mathbb{T}_{04}^{5} \quad: \quad\left(\mathbb{T}_{01}^{3}\right)_{5,1} \quad: \quad e_{1} e_{2}=e_{3}, \quad e_{1} e_{3}=e_{4}, \quad e_{2} e_{3}=e_{5} .
\end{aligned}
$$

\subsection{The algebraic classification of 5-dimensional nilpotent Tortkara algebras}

The algebraic classification of 4-dimensional nilpotent Tortkara algebras

\begin{tabular}{|l|l|l|l|l|}
\hline $\mathbf{A}$ & multiplication tables & $H^{2}(\mathbf{A})$ & Automorphisms \\
\hline \hline \multirow{2}{*}{$\mathbb{T}_{01}^{4}$} & $e_{1} e_{2}=e_{3}$ & {$\left[\Delta_{13}\right],\left[\Delta_{14}\right],\left[\Delta_{23}\right],\left[\Delta_{24}\right],\left[\Delta_{34}\right]$} & $\left(\begin{array}{llll}x & y & 0 & 0 \\
z & u & 0 & 0 \\
v & h & x u-z y & g \\
l & r & 0 & t\end{array}\right)$ \\
\hline \multirow{2}{*}{$\mathbb{T}_{02}^{4}$} & $e_{1} e_{2}=e_{3}, e_{1} e_{3}=e_{4}$ & {$\left[\Delta_{14}\right],\left[\Delta_{23}\right],\left[\Delta_{24}\right]$} & $\left(\begin{array}{cccc}x & 0 & 0 & 0 \\
y & z & 0 & 0 \\
u & v & x z & 0 \\
h & g & x v & x^{2} z\end{array}\right)$ \\
\hline
\end{tabular}

1-dimensional central extensions of $\mathfrak{N}_{4}$

Thanks to [41], we have all non-split 1-dimensional anticommutative central extensions of $\mathfrak{N}_{4}$ :

$\mathbb{T}_{05}^{5} \quad: \quad\left(\mathfrak{N}_{4}\right)_{5,1} \quad: \quad e_{1} e_{2}=e_{5}, \quad e_{3} e_{4}=e_{5}$

\section{1-dimenisonal central extensions of $\mathbb{T}_{01}^{4}$}

Since

$$
\left(\begin{array}{ccccc}
x & y & 0 & 0 \\
z & u & 0 & 0 \\
v & h & x u-z y & g \\
l & r & 0 & t
\end{array}\right)^{t}\left(\begin{array}{cccc}
0 & 0 & \alpha_{1} & \alpha_{2} \\
0 & 0 & \alpha_{3} & \alpha_{4} \\
-\alpha_{1} & -\alpha_{3} & 0 & \alpha_{5} \\
-\alpha_{2} & -\alpha_{4} & -\alpha_{5} & 0
\end{array}\right)\left(\begin{array}{cccc}
x & y & 0 & 0 \\
z & u & 0 & 0 \\
v & h & x u-z y & g \\
l & r & 0 & t
\end{array}\right)=\left(\begin{array}{cccc}
0 & \alpha^{*} & \alpha_{1}^{*} & \alpha_{2}^{*} \\
-\alpha^{*} & 0 & \alpha_{3}^{*} & \alpha_{4}^{*} \\
-\alpha_{1}^{*} & -\alpha_{3}^{*} & 0 & \alpha_{5}^{*} \\
-\alpha_{2}^{*} & -\alpha_{4}^{*} & -\alpha_{5}^{*} & 0
\end{array}\right)
$$

where

$$
\begin{aligned}
\alpha^{*} & =\left(\alpha_{1} x+\alpha_{3} z-\alpha_{5} l\right) h+\left(-\alpha_{2} y-\alpha_{4} u\right) l+\left(\alpha_{2} x+\alpha_{4} z+\alpha_{5} v\right) r-v\left(\alpha_{1} y+\alpha_{3} u\right) \\
\alpha_{1}^{*} & =\left(\alpha_{1} x+\alpha_{3} z-\alpha_{5} l\right)(u x-y z) \\
\alpha_{2}^{*} & =\left(\alpha_{1} x+\alpha_{3} z-\alpha_{5} l\right) g+\left(\alpha_{2} x+\alpha_{4} z+\alpha_{5} v\right) t \\
\alpha_{3}^{*} & =\left(\alpha_{1} y+\alpha_{3} u-\alpha_{5} r\right)(u x-y z) \\
\alpha_{4}^{*} & =\left(\alpha_{1} y+\alpha_{3} u-\alpha_{5} r\right) g+\left(\alpha_{2} y+\alpha_{4} u+\alpha_{5} h\right) t \\
\alpha_{5}^{*} & =(u x-y z) \alpha_{5} t
\end{aligned}
$$


we obtain that the action of Aut $\left(\mathbb{T}_{01}^{4}\right)$ on a subspace

$$
\left\langle\alpha_{1}\left[\Delta_{13}\right]+\alpha_{2}\left[\Delta_{14}\right]+\alpha_{3}\left[\Delta_{23}\right]+\alpha_{4}\left[\Delta_{24}\right]+\alpha_{5}\left[\Delta_{34}\right]\right\rangle
$$

is given by

$$
\left\langle\alpha_{1}^{*}\left[\Delta_{13}\right]+\alpha_{2}^{*}\left[\Delta_{14}\right]+\alpha_{3}^{*}\left[\Delta_{23}\right]+\alpha_{4}^{*}\left[\Delta_{24}\right]+\alpha_{5}^{*}\left[\Delta_{34}\right]\right\rangle .
$$

We provide the orbit of every possible case:

1. If $\alpha_{5} \neq 0$, then choosing $g=0, l=-\frac{\alpha_{1} x+\alpha_{3} z}{\alpha_{5}}, v=-\frac{\alpha_{2} x+\alpha_{4} z}{\alpha_{5}}, r=-\frac{\alpha_{1} y+\alpha_{3} u}{\alpha_{5}}$, $h=-\frac{\alpha_{2} y+\alpha_{4} u}{\alpha_{5}}$, we have a representative $\left\{(u x-y z) t \alpha_{5}\left[\Delta_{34}\right]\right\}$, and the orbit is $\left\langle\left[\Delta_{34}\right]\right\rangle$.

2. If $\alpha_{5}=0, \alpha_{1}=\alpha_{3}=0$, then we can assume that $\alpha_{2} \neq 0$ and $\alpha_{4} \neq 0$, and, choosing $u=-\frac{\alpha_{2} y}{\alpha_{4}}$, we have a representative $\left\{\left(\alpha_{2} x+\alpha_{4} z\right) t\left[\Delta_{14}\right]\right\}$, and the orbit is $\left\langle\left[\Delta_{14}\right]\right\rangle$.

3. If $\alpha_{5}=0$ and $\alpha_{1} \neq 0$ or $\alpha_{3} \neq 0$, we can assume that $\alpha_{1} \neq 0$ and $\alpha_{3} \neq 0$. Then

(a) if $\alpha_{2} \alpha_{3}=\alpha_{1} \alpha_{4}$, then choosing $g=-\frac{\alpha_{4}}{\alpha_{3}} t$ and $x=y+\frac{\alpha_{3}}{\alpha_{1}}(u-z)$ we have a representative $\left\{\left(\alpha_{1} x+\alpha_{3} z\right)(u x-y z)\left(\left[\Delta_{13}\right]+\left[\Delta_{23}\right]\right)\right\}$ and the orbit is $\left\langle\left[\Delta_{13}\right]+\left[\Delta_{23}\right]\right\rangle$.

(b) if $\alpha_{2} \alpha_{3} \neq \alpha_{1} \alpha_{4}$, then we can suppose that $\alpha_{4} \neq 0$. Choosing $y=\sqrt{-\frac{\alpha_{4}^{2} x t}{\alpha_{3}\left(\alpha_{2} x+\alpha_{4} t\right)}}$, $z=-\frac{\alpha_{1} x}{\alpha_{3}}, u=-\frac{\alpha_{2} y}{\alpha_{4}}$ we have a representative $\left\{\left(\alpha_{1} y+\alpha_{3} u\right)(u x-y z)\left(\left[\Delta_{14}\right]+\left[\Delta_{23}\right]\right)\right\}$ and the orbit is $\left\langle\left[\Delta_{14}\right]+\left[\Delta_{23}\right]\right\rangle$.

It is easy to verify that all the previous orbits are different so that we arrive at

$$
T_{1}\left(\mathbb{T}_{01}^{4}\right)=\left\langle\left[\Delta_{13}\right]+\left[\Delta_{23}\right]\right\rangle \cup\left\langle\left[\Delta_{14}\right]\right\rangle \cup\left\langle\left[\Delta_{14}\right]+\left[\Delta_{23}\right]\right\rangle \cup\left\langle\left[\Delta_{34}\right]\right\rangle .
$$

Note that the orbit $\left\langle\left[\Delta_{13}\right]+\left[\Delta_{23}\right]\right\rangle$ gives a split central extension and the orbit $\left\langle\left[\Delta_{14}\right]\right\rangle$ gives a non-split central extension with 2-dimensional annihilator (it is a 2-dimensional central extension of a 3-dimensional algebra). Now we have all non-split 1-dimensional central extensions with 1dimensional annihilator of $\mathbb{T}_{01}^{4}$ :

$$
\begin{aligned}
& \mathbb{T}_{06}^{5}:\left(\mathbb{T}_{01}^{4}\right)_{5,1} \quad: \quad e_{1} e_{2}=e_{3}, \quad e_{1} e_{4}=e_{5}, \quad e_{2} e_{3}=e_{5}, \\
& \mathbb{T}_{07}^{5}:\left(\mathbb{T}_{01}^{4}\right)_{5,2}: e_{1} e_{2}=e_{3}, \quad e_{3} e_{4}=e_{5} .
\end{aligned}
$$

\section{1-dimensional central extensions of $\mathbb{T}_{02}^{4}$}

Since

$$
\left(\begin{array}{cccc}
0 & \alpha^{*} & \alpha^{* *} & \alpha_{1}^{*} \\
-\alpha^{*} & 0 & \alpha_{2}^{*} & \alpha_{3}^{*} \\
-\alpha^{* *} & -\alpha_{2}^{*} & 0 & 0 \\
-\alpha_{1}^{*} & -\alpha_{3}^{*} & 0 & 0
\end{array}\right)=\left(\begin{array}{cccc}
x & 0 & 0 & 0 \\
y & z & 0 & 0 \\
u & v & x z & 0 \\
h & g & x v & x^{2} z
\end{array}\right)^{t}\left(\begin{array}{cccc}
0 & 0 & 0 & \alpha_{1} \\
0 & 0 & \alpha_{2} & \alpha_{3} \\
0 & -\alpha_{2} & 0 & 0 \\
-\alpha_{1} & -\alpha_{3} & 0 & 0
\end{array}\right)\left(\begin{array}{cccc}
x & 0 & 0 & 0 \\
y & z & 0 & 0 \\
u & v & x z & 0 \\
h & g & x v & x^{2} z
\end{array}\right)
$$

with

$$
\begin{aligned}
\alpha^{*} & =\left(-\alpha_{2} u-\alpha_{3} h\right) z+\alpha_{3} g y+\alpha_{1} g x+\alpha_{2} y v \\
\alpha^{* *} & =\left(\alpha_{2} z+\alpha_{3} v\right) y x+\alpha_{1} v^{2}, \\
\alpha_{1}^{*} & =\left(\alpha_{1} x+\alpha_{3} y\right) x^{2} z, \\
\alpha_{2}^{*} & =\left(\alpha_{2} z+\alpha_{3} v\right) x z \\
\alpha_{3}^{*} & =\alpha_{3} z^{2} x^{2}
\end{aligned}
$$


we obtain that the action of Aut $\left(\mathbb{T}_{2}^{4}\right)$ on a subspace $\left\langle\alpha_{1}\left[\Delta_{14}\right]+\alpha_{2}\left[\Delta_{23}\right]+\alpha_{3}\left[\Delta_{24}\right]\right\rangle$ is given by

$$
\left\langle\alpha_{1}^{*}\left[\Delta_{14}\right]+\alpha_{2}^{*}\left[\Delta_{23}\right]+\alpha_{3}^{*}\left[\Delta_{24}\right]\right\rangle .
$$

We provide the orbit of every possible case:

1. If $\alpha_{5} \neq 0$, then choosing $x=1, z=\frac{1}{\sqrt{\alpha_{3}}}, y=-\frac{\alpha_{1}}{\alpha_{3}}, v=-\frac{\alpha_{2}}{\sqrt{\left(\alpha_{3}\right)^{3}}}$ we have the orbit is $\left\langle\left[\Delta_{24}\right]\right\rangle$.

2. If $\alpha_{5}=0$, then we have one from the following orbits $\left\langle\left[\Delta_{14}\right]\right\rangle,\left\langle\left[\Delta_{14}\right]+\left[\Delta_{23}\right]\right\rangle,\left\langle\left[\Delta_{23}\right]\right\rangle$.

It is easy to verify that all the previous orbits are different so that we arrive at

$$
T_{1}\left(\mathbb{T}_{02}^{4}\right)=\left\langle\left[\Delta_{14}\right]\right\rangle \cup\left\langle\left[\Delta_{14}\right]+\left[\Delta_{23}\right]\right\rangle \cup\left\langle\left[\Delta_{23}\right]\right\rangle \cup\left\langle\left[\Delta_{24}\right]\right\rangle .
$$

We therefore have all non-split 1-dimensional central extensions with 1-dimensional annihilator of $\mathbb{T}_{02}^{4}$ :

$$
\begin{aligned}
& \mathbb{T}_{08}^{5} \quad: \quad\left(\mathbb{T}_{02}^{4}\right)_{5,1} \quad: \quad e_{1} e_{2}=e_{3}, \quad e_{1} e_{3}=e_{4}, \quad e_{1} e_{4}=e_{5}, \\
& \mathbb{T}_{09}^{5}:\left(\mathbb{T}_{02}^{4}\right)_{5,2} \quad: \quad e_{1} e_{2}=e_{3}, \quad e_{1} e_{3}=e_{4}, \quad e_{1} e_{4}=e_{5}, \quad e_{2} e_{3}=e_{5} \text {, } \\
& \mathbb{T}_{10}^{5}:\left(\mathbb{T}_{02}^{4}\right)_{5,3} \quad: \quad e_{1} e_{2}=e_{3}, \quad e_{1} e_{3}=e_{4}, \quad e_{2} e_{4}=e_{5} .
\end{aligned}
$$

The algebraic classification of 5-dimesional nilpotent Tortkara algebras

Theorem 1.1. Let $\mathbf{A}$ be a nontrivial 5-dimensional nilpotent Tortkara algebra. Then, A is isomorphic to exactly one of the following algebras:

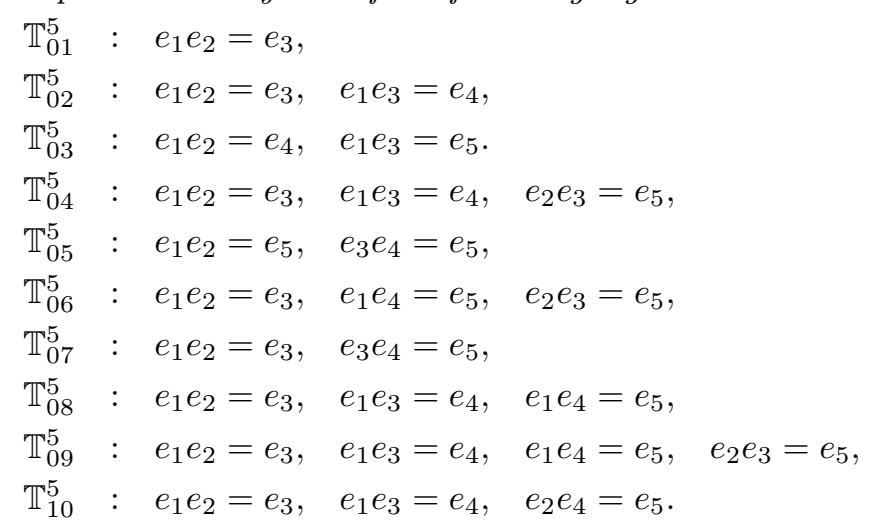

\section{The geometric classification of 5-dimesional nilpotent Tortkara algebras}

\subsection{Preliminaries}

\section{Definitions and notation}

Given an $n$-dimensional vector space $\mathbb{V}$, the set $\operatorname{Hom}(\mathbb{V} \otimes \mathbb{V}, \mathbb{V}) \cong \mathbb{V}^{*} \otimes \mathbb{V}^{*} \otimes \mathbb{V}$ is a vector space of dimension $n^{3}$. This space has a structure of the affine variety $\mathbb{C}^{n^{3}}$. Indeed, fix a basis $e_{1}, \ldots, e_{n}$ of $\mathbb{V}$. Then, any $\mu \in \operatorname{Hom}(\mathbb{V} \otimes \mathbb{V}, \mathbb{V})$ is determined by $n^{3}$ structure constants $c_{i, j}^{k} \in \mathbb{C}$ such that $\mu\left(e_{i} \otimes e_{j}\right)=\sum_{k=1}^{n} c_{i, j}^{k} e_{k}$. A subset of $\operatorname{Hom}(\mathbb{V} \otimes \mathbb{V}, \mathbb{V})$ is Zariski-closed if it can be defined by a set of polynomial equations in the variables $c_{i, j}^{k}(1 \leqslant i, j, k \leqslant n)$. 
Let $T$ be a set of polynomial identities. All algebra structures on $\mathbb{V}$ satisfying polynomial identities from $T$ form a Zariski-closed subset of the variety $\operatorname{Hom}(\mathbb{V} \otimes \mathbb{V}, \mathbb{V})$. We denote this subset by $\mathbb{L}(T)$. The general linear group $G L(\mathbb{V})$ acts on $\mathbb{L}(T)$ by conjugations:

$$
(g * \mu)(x \otimes y)=g \mu\left(g^{-1} x \otimes g^{-1} y\right)
$$

for $x, y \in \mathbb{V}, \mu \in \mathbb{L}(T) \subset \operatorname{Hom}(\mathbb{V} \otimes \mathbb{V}, \mathbb{V})$ and $g \in G L(\mathbb{V})$. Thus, $\mathbb{L}(T)$ is decomposed into $G L(\mathbb{V})$-orbits that correspond to isomorphism classes of algebras. Let $O(\mu)$ denote the orbit of $\mu \in \mathbb{L}(T)$ under the action of $G L(\mathbb{V})$ and $\overline{O(\mu)}$ denote the Zariski closure of $O(\mu)$.

Let $A$ and $B$ be two $n$-dimensional algebras satisfying identities from $T$ and $\mu, \lambda \in \mathbb{L}(T)$ represent $A$ and $B$, respectively. We say that $A$ degenerates to $B$ and denote $A \rightarrow B$ if $\lambda \in \overline{O(\mu)}$. Note that, in this case, we have $\overline{O(\lambda)} \subset \overline{O(\mu)}$. Hence, the definition of a degeneration does not depend on the choice of $\mu$ and $\lambda$. If $A \neq B$, then the assertion $A \rightarrow B$ is called a proper degeneration. We write $A \nrightarrow \rightarrow B$ if $\lambda \notin \overline{O(\mu)}$.

Let $A$ be represented by $\mu \in \mathbb{L}(T)$. Then $A$ is rigid in $\mathbb{L}(T)$ if $O(\mu)$ is an open subset of $\mathbb{L}(T)$. Recall that a subset of a variety is called irreducible if it cannot be represented as a union of two non-trivial closed subsets. A maximal irreducible closed subset of a variety is called an irreducible component. It is well known that any affine variety can be represented as a finite union of its irreducible components in a unique way. The algebra $A$ is rigid in $\mathbb{L}(T)$ if and only if $\overline{O(\mu)}$ is an irreducible component of $\mathbb{L}(T)$.

Given spaces $U$ and $W$, we write simply $U>W$ instead of $\operatorname{dim} U>\operatorname{dim} W$.

\section{A method for description of all degenerations of algebras}

In the present work we use the methods from $[12,29,30,41]$ applied to Lie algebras. First of all, if $A \rightarrow B$ and $A \neq B$, then $\mathfrak{D e r}(A)<\mathfrak{D e r}(B)$, where $\mathfrak{D e r}(A)$ is the Lie algebra of derivations of $A$. We will compute the dimensions of algebras of derivations and will check the assertion $A \rightarrow B$ only for such $A$ and $B$ that $\mathfrak{D e r}(A)<\mathfrak{D e r}(B)$. Secondly, if $A \rightarrow C$ and $C \rightarrow B$ then $A \rightarrow B$. If there is no $C$ such that $A \rightarrow C$ and $C \rightarrow B$ are proper degenerations, then the assertion $A \rightarrow B$ is called a primary degeneration. If $\mathfrak{D e r}(A)<\mathfrak{D e r}(B)$ and there are no $C$ and $D$ such that $C \rightarrow A, B \rightarrow D, C \nrightarrow \rightarrow D$ and one of the assertions $C \rightarrow A$ and $B \rightarrow D$ is a proper degeneration, then the assertion $A \nrightarrow \rightarrow B$ is called a primary non-degeneration. It suffices to prove only primary degenerations and non-degenerations to describe degenerations in the variety under consideration. It is easy to see that any algebra degenerates to the algebra with zero multiplication. From now on, we use this fact without mentioning it.

To prove primary degenerations, we construct families of matrices parametrized by $t$. Namely, let $A$ and $B$ be two algebras represented by the structures $\mu$ and $\lambda$ from $\mathbb{L}(T)$, respectively. Let $e_{1}, \ldots, e_{n}$ be a basis of $\mathbb{V}$ and $c_{i, j}^{k}(1 \leqslant i, j, k \leqslant n)$ be the structure constants of $\lambda$ in this basis. If there exist $a_{i}^{j}(t) \in \mathbb{C}\left(1 \leqslant i, j \leqslant n, t \in \mathbb{C}^{*}\right)$ such that $E_{i}^{t}=\sum_{j=1}^{n} a_{i}^{j}(t) e_{j}(1 \leqslant i \leqslant n)$ form a basis of $\mathbb{V}$ for any $t \in \mathbb{C}^{*}$, and the structure constants of $\mu$ in the basis $E_{1}^{t}, \ldots, E_{n}^{t}$ are such polynomials $c_{i, j}^{k}(t) \in \mathbb{C}[t]$ that $c_{i, j}^{k}(0)=c_{i, j}^{k}$, then $A \rightarrow B$. In this case, $E_{1}^{t}, \ldots, E_{n}^{t}$ is called a parametrized basis for $A \rightarrow B$.

\subsection{The geometric classification of 5-dimensional nilpotent Tortkara algebras}

The main result of the present section is the following theorem.

Theorem 2.1. The variety of 5-dimensional nilpotent Tortkara algebras has one irreducible component defined by the rigid algebra $\mathbb{T}_{10}^{5}$. The graph of primary degenerations for 5 -dimensional nilpotent Tortkara algebras has the following form: 


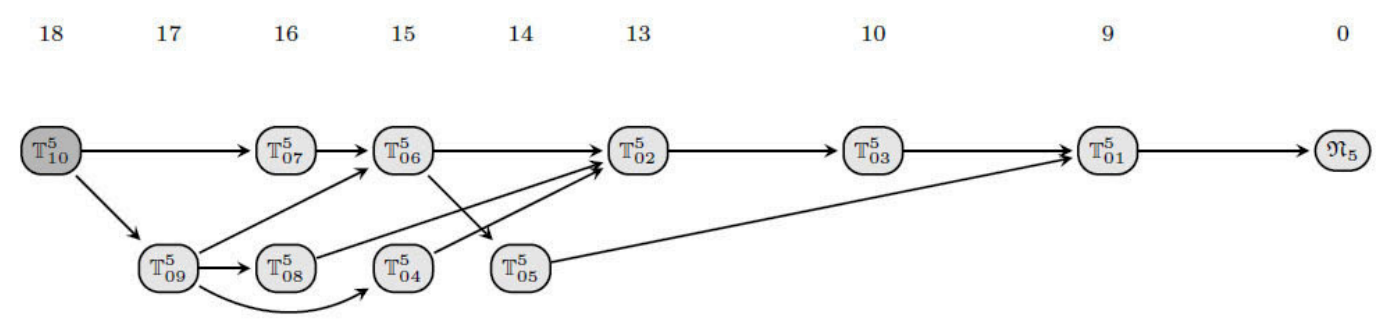

Proof. Note that the set $\left\{\mathbb{T}_{0 i}^{5}\right\}_{1 \leqslant i \leqslant 9} \cup\left\{\mathfrak{N}_{5}\right\}$ gives the variety of all 5-dimensional nilpotent Malcev algebras. The descrition of all degenerations of 5-dimensional nilpotent Malcev algebras was obtained in [36]. By calculation of the dimension of the algebra of derivations of $\mathbb{T}_{10}^{5}$, we have the dimension of the orbit closure of $\mathbb{T}_{10}^{5}$.

The parametrized basis formed by

$$
E_{1}^{t}=t e_{1}+\frac{1}{t^{2}} e_{2}, E_{2}^{t}=t e_{2}+\frac{1}{t} e_{3}, E_{3}^{t}=t^{2} e_{3}+e_{4}, E_{4}^{t}=t^{3} e_{4}+\frac{1}{t^{2}} e_{5}, E_{5}^{t}=t e_{5}
$$

gives the degeneration $\mathbb{T}_{10}^{5} \rightarrow \mathbb{T}_{09}^{5}$.

The parametrized basis formed by

$$
E_{1}^{t}=e_{1}, E_{2}^{t}=e_{3}, E_{3}^{t}=e_{4}, E_{4}^{t}=t e_{2}, E_{5}^{t}=-t e_{5}
$$

gives the degeneration $\mathbb{T}_{10}^{5} \rightarrow \mathbb{T}_{07}^{5}$. Thus, the theorem is proved.

The first part of this work is supported by the Russian Science Foundation under grant 18-711000\%. The second part of this work was supported by UAEU UPAR (9) 2017 Grant G00002599 (Mohamed A.Salim).

\section{References}

[1] J.Adashev, L.Camacho, S.Gomez-Vidal, I.Karimjanov, Naturally graded Zinbiel algebras with nilindex $n-3$, Linear Algebra Appl., 443(2014), 86-104.

[2] J.Adashev, L.Camacho, B.Omirov, Central extensions of null-filiform and naturally graded filiform non-Lie Leibniz algebras, J. Algebra, 479(2017), 461-486.

[3] M.A.Alvarez, On rigid 2-step nilpotent Lie algebras, Algebra Colloquium, 25(2018), no. 2, 349-360.

[4] M.A.Alvarez, The variety of 7-dimensional 2-step nilpotent Lie algebras, Symmetry, 10(2018), no. 1, 26.

[5] M.A.Alvarez, I.Hernández, On degenerations of Lie superalgebras, Linear and Multilinear Algebra, DOI: 10.1080/03081087.2018.1498060.

[6] M.A.Alvarez, I.Hernández, I.Kaygorodov, Degenerations of Jordan superalgebras, Bulletin of the Malaysian Mathematical Sciences Society, 2018, DOI: 10.1007/s40840-018-0664-3.

[7] G.G.A.Bauerle, E.A.de Kerf, A.P.E.ten Kroode, Lie Algebras. Part 2, Finite and Infinite Dimensional Lie Algebras and Applications in Physics, edited and with a preface by E.M. de Jager, Studies in Mathematical Physics, vol. 7, North-Holland Publishing Co., Amsterdam, 1997. 
[8] T.Benes, D.Burde, Degenerations of pre-Lie algebras, Journal of Mathematical Physics, 50(2009), no. 11, 112102.

[9] T.Benes, D.Burde, Classification of orbit closures in the variety of three dimensional Novikov algebras, Journal of Algebra and Its Applications, 13(2014), no. 2, 1350081.

[10] M.Bremner, On Tortkara triple systems, Communications in Algebra, 46(2018), no. 6, 2396-2404.

[11] D.Burde, Degenerations of nilpotent Lie algebras, Journal of Lie Theory, 9(1999), no. 1, 193-202.

[12] D.Burde, C.Steinhoff, Classification of orbit closures of 4-dimensional complex Lie algebras, Journal of Algebra, 214(1999), no. 2, 729-739.

[13] A.Calderón Martín, A.Fernández Ouaridi, I.Kaygorodov, The classification of $n$-dimensional anticommutative algebras with $(n-3)$-dimensional annihilator, Communications in Algebra 2018, DOI:10.1080/00927872.2018.1468909.

[14] A.Calderón Martín, A.Fernández Ouaridi, I.Kaygorodov, The classification of bilinear maps with radical of codimension 2, arXiv:1806.07009.

[15] L.Camacho, E.Cañete, S.Gomez-Vidal, B.Omirov, $p$-filiform Zinbiel algebras, Linear Algebra Appl., 438(2013), no. 7, 2958-2972.

[16] S.Cicalo, W.De Graaf, C.Schneider, Six-dimensional nilpotent Lie algebras, Linear Algebra Appl., 436(2012), no. 1, 163-189.

[17] I.Darijani, H.Usefi, The classification of 5-dimensional p-nilpotent restricted Lie algebras over perfect fields, I., J. Algebra, 464(2016), 97-140.

[18] W.De Graaf, Classification of nilpotent associative algebras of small dimension, Internat. J. Algebra Comput., 28(2018), no. 1, 133-161.

[19] W.De Graaf, Classification of 6-dimensional nilpotent Lie algebras over fields of characteristic not 2, J. Algebra, 309 (2007), no. 2, 640-653.

[20] I.Dokas, Zinbiel algebras and commutative algebras with divided powers, Glasg. Math. J., 52(2010), no. 2, 303-313.

[21] A.Dzhumadildaev, Zinbiel algebras under q-commutators, Journal of Mathematical Sciences (New York), 144(2007), no. 2, 3909-3925.

[22] A.Dzhumadildaev, K.Tulenbaev, Nilpotency of Zinbiel algebras, J. Dyn. Control Syst., 11(2005), no. 2, 195-213.

[23] A.Dzhumadildaev, N.Ismailov, F.Mashurov, Embeddable algebras into Zinbiel algebras via the commutator, arXiv:1809.10550.

[24] A.Hegazi, H.Abdelwahab, Nilpotent evolution algebras over arbitrary fields, Linear Algebra Appl., 486(2015), 345-360.

[25] A.Hegazi, H.Abdelwahab, Classification of five-dimensional nilpotent Jordan algebras, Linear Algebra and its Applications, 494(2016), 165-218.

[26] A.Hegazi, H.Abdelwahab, The classification of $n$-dimensional non-associative Jordan algebras with $(n-3)$-dimensional annihilator, Communications in Algebra, 46(2018), no. 2, 629-643. 
[27] A.Hegazi, H.Abdelwahab, A.Calderón Martín, The classification of $n$-dimensional non-Lie Malcev algebras with $(n-4)$-dimensional annihilator, Linear Algebra Appl., 505(2016), $32-56$.

[28] A.Hegazi, H.Abdelwahab, A.Calderón Martín, Classification of nilpotent Malcev algebras of small dimensions over arbitrary fields of characteristic not 2, Algebr. Represent. Theory, $\mathbf{2 1}(2018)$, no. 1, 19-45.

[29] F.Grunewald, J.O'Halloran, Varieties of nilpotent Lie algebras of dimension less than six, Journal of Algebra, 112 (1988), 315-325.

[30] F.Grunewald, J.O'Halloran, A Characterization of orbit closure and applications, Journal of Algebra, 116(1988), 163-175.

[31] F.Grunewald, J.O'Halloran, Deformations of Lie algebras, Journal of Algebra, 162(1993), no. 1, 210-224.

[32] N.Ismailov, I.Kaygorodov, Yu.Volkov, The geometric classification of Leibniz algebras, International Journal of Mathematics, 29(2018), no. 5, 1850035.

[33] N.Ismailov, I.Kaygorodov, Yu.Volkov, Degenerations of Leibniz and anticommutative algebras, arXiv:1808.00907.

[34] I.Karimjanov, I.Kaygorodov, M.Ladra, Central extensions of filiform associative algebras, arXiv:1809.00183.

[35] I.Kaygorodov, Yu.Popov, A.Pozhidaev, Yu.Volkov, Degenerations of Zinbiel and nilpotent Leibniz algebras, Linear and Multilinear Algebra, 66(2018), no. 4, 704-716.

[36] I.Kaygorodov, Yu.Popov, Yu.Volkov, Degenerations of binary-Lie and nilpotent Malcev algebras, Communications in Algebra, 46(2018), no. 11, 4929-4941.

[37] I.Kaygorodov, Yu.Volkov, The variety of 2-dimensional algebras over an algebraically closed field, Canadian Journal of Mathematics, DOI: 10.4153/S0008414X18000056.

[38] I. Kaygorodov, Yu. Volkov, The degeneration level classification of algebras, arXiv:1710.08943.

[39] J.-L.Loday, Cup-product for Leibniz cohomology and dual Leibniz algebras, Math. Scand., 77(1995), no. 2, 189-196.

[40] A.Naurazbekova, U.Umirbaev, Identities of dual Leibniz algebras, TWMS J. Pure Appl. Math., 1(2010), no. 1, 86-91.

[41] C.Seeley, Degenerations of 6-dimensional nilpotent Lie algebras over $\mathbb{C}$, Communications in Algebra, 18(1990), 3493-3505.

[42] T.Skjelbred, T.Sund, Sur la classification des algebres de Lie nilpotentes, C. R. Acad. Sci. Paris Ser. A-B, 286(1978), no. 5, 241-242.

[43] I.Rakhimov, M.Hassan, On one-dimensional Leibniz central extensions of a filiform Lie algebra, Bull. Aust. Math. Soc., 84(2011), no. 2, 205-224.

[44] D.Yau, Deformation of dual Leibniz algebra morphisms, Comm. Algebra, 35(2007), no. 4, 1369-1378.

[45] P.Zusmanovich, Central extensions of current algebras, Trans. Amer. Math. Soc., 334(1992), no. 1, 143-152. 


\section{Многоообразие нильпотентных алгебр Торткара}

Илья Б. Горшков

Институт математики и фундаментальной информатики

Сибирский федеральный университет

Свободный, 79, Красноярск, 660041

Россия

Иван Кайгородов

CMCC, Федеральный университет АВС

Санто Андре, Бразилия

Алексей А. Кытманов

Институт космических и информационных технологий

Сибирский федеральный университет

Киренского, 26, Красноярск, 660074

Россия

Мохамед А. Салим

Университет Объединенных Арабских Эмиратов Аль-Айн, ОАЭ

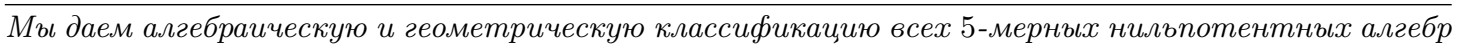
Торткара над комплексным полем.

Ключевые слова: алгебра Торткара, алгебраическая классификачия, чентральное расширение, геометрическая классификачия, вырождения. 